\title{
EFFECT OF FUNCTIONAL MOTION IMAGE ANALYSIS UNDER MULTI-LAYER CONVOLUTIONAL NEURAL NETWORK ON IMPROVING SPEED AND FORCE
}

\author{
Jerry long, Macmillan Thomas \\ Centre for Sport Research, School of Exercise and Nutrition Sciences, Deakin University, Geelong, Australia. \\ *Corresponding Author Email:
}

This is an open access article distributed under the Creative Commons Attribution License, which permits unrestricted use, distribution, and reproduction in any medium, provided the original work is properly cited

\section{ARTICLE DETAILS}

\section{Article History:}

Received 16 November 2019 Accepted 20 Decmber 2019 Available online 8 January 2020

\section{ABSTRACT}

To explore the effect of applying multi-layer convolutional neural network to noise removal during the acquisition and transmission of functional motion images, the classical image denoising algorithms of mean filtering, median filtering, and wavelet transform filtering are first introduced. In addition, the evaluation methods of mean square error (MSE), image enhancement factor (IEF), peak signal to noise ratio (PSNR) and structural similarity measure (SSIM) in the image quality evaluation index system are introduced. Based on the convolutional neural network model, a multi-scale parallel convolutional neural network (MP-CNN) model is constructed to remove the noise in the image, and the functional action image is devoted to different degrees. Finally, the denoising effect is evaluated by the objective and subjective evaluation system. The objective evaluation results show that MP-CNN's MSE, IEF, PSNR, and SSIM are better than the single-channel model, and the test time is shorter. The subjective evaluation results show that the MP-CNN model has the best effect on noise removal after 25 denoising of functional action images. In this study, a multi-channel image denoising model based on the multi-layer convolutional neural network can improve the effect of functional motion image noise removal.

\section{KEYWORDS}

Multi-layer convolutional neural network, Functional action-image, Noise, Evaluation system

\section{INTRODUCTION}

People's access to external information usually comes from the visual system, which indicates that image information is a very important means for human to extract important information. In recent years, information technology and intelligent technology show a trend of rapid development. More and more videos, animations, photos and other information are widely used in people's daily life. With the maturity of digital imaging technology, digital image processing and analysis become easier. According to the survey results, about $75 \%$ or more of the information obtained by human comes from the visual system, while 85 or more of the external information received by the human brain comes from the visual system. The image is a real response to objective things, but noise is inevitable in the process of image acquisition and transmission, which is caused by sensor quality, external environment and interference of transmission channel. In order to better apply the image to various fields, the image needs to be denoised, segmented, fused and feature detection, among which image denoising is the most critical step.

At present, there are more and more researches on the application of the convolutional neural network in image denoising. A study showed that image denoising is a basic problem in computer vision and image processing, and it used the glassman manifold algorithm to remove image noise and improve the visual quality [1]. A group researcher used realtime image noise filter to reduce the noise of MRI images and found that they could improve the signal-to-noise ratio by $60 \%$, without introducing image artifacts and prolonging the detection time [2]. In other study, they used the new deep residual convolutional neural network for image denoising, and the results showed that its denoising effect was better than other algorithms [3]. Some of researchers proposed a pulse-coupled neural network with multi-channel link and feed field, which can be used in satellite image segmentation to improve the processing speed [4]. In other hand, researchers showed that deep convolutional neural network can significantly improve image denoising, but it lacks fine high-frequency detail processing [5]. Some of them also applied the convolutional neural network to the noise removal of 3D medical imaging images and found that it could effectively suppress the noise and retain important feature structures [6].

To sum up, convolutional neural network has been widely used in image noise processing, but its application in the analysis of functional action images is relatively rare. Therefore, based on the multi-layer convolutional neural network model, it is applied to the noise removal of functional action images, and the effect of constructing the denoising model through the evaluation index of image quality is detected. The purpose of this study is to lay a foundation for improving the quality of acquisition and transmission of functional action images.

\section{METHODOLOGY}

\section{$2.1 \quad$ Image denoising algorithm}

Noise generally has the characteristics of random distribution, and the probability density function can be used to describe the noise distribution, which can be divided into Gaussian, Rayleigh, exponent, $\gamma$, uniform and impulse noise and so on. The classical denoising methods include mean filtering, median filtering and wavelet transform filtering. Mean filtering is a common spatial linear filtering method, which plays an obvious role in smoothing noise, and the Box template is often used to filter noise images. 
Then, the mean value calculation formula of pixel values in the template is as follows:

$$
z(x, y)=\frac{1}{m \cdot n} \sum_{(i, j) \in \Omega_{x y}} g(i, j)
$$

In equation $1, G(i, j)$ represents the original pixel value at the position $(i, j)$, $\mathrm{z}(\mathrm{x}, \mathrm{y})$ represents the pixel value after the mean value is assigned, $\Omega_{\mathrm{xy}}$ is for $(x, y)$ point and the set of pixel values in rectangular areas of the size of $m$. n.

Median filtering is one of the more famous denoising algorithms, which also requires the removal of image noise based on domain operation. Its calculation formula is as follows:

$$
g(x, y)=\text { median }[f(x-i, y-j)],(x, y) \in S
$$

In equation $2, g(x, y)$ and $f(x, y)$ is the gray value of the pixel, and $S$ is the template window. The wavelet transformation filtering algorithm first needs to carry out the wavelet transform for the image with noise. After finding and setting the appropriate threshold value, the wavelet coefficient is selected for the image signal and noise signal. Finally, the retained wavelet coefficients are inversely converted. The calculation process is as follows: image with noise, multi-scale decomposition, de-noising processing of each scale, inverse calculation of wavelet coefficient, and image with noise removed.

\subsection{Evaluation method of image quality after denoising}

Image evaluation methods can be divided into the subjective evaluation and objective evaluation. Although the subjective evaluation can reflect the image quality more truly, the operation method is more complicated, and the mathematical model can't be established, so the application scope of this method is limited. Objective evaluation is to construct a mathematical model related to image quality based on the working principle of the visual system of human eyes, which can quantitatively reflect the image quality. Common methods include MSE, IEF, PSNR and SSIM. MSE can reflect the distortion degree of images before and after denoising, and its calculation formula is as follows:

$M S E=\frac{1}{m \cdot n} \sum_{i=1}^{m} \sum_{j=1}^{n}(\hat{f}(i, j)-f(i, j))^{2}$

In equation $3, f(i, j)$ represents the pixel value at $(\mathrm{i}, \mathrm{j})$ of the original image without noise, $\hat{f}(i, j)$ represents the pixel value at $(\mathrm{i}, \mathrm{j})$ after denoising, and $\mathrm{m}$ and $\mathrm{n}$ are the length and width of the image. IEF is used to reflect the protection of image edge performance by image denoising algorithm, and its calculation equation is as follows:

$$
I E F=\left\{\sum_{i=1}^{m} \sum_{j=1}^{n}\left(f(i, j)-\mu_{0}(i, j)\right)^{2}\right\} /\left\{\sum_{i=1}^{m} \sum_{j=1}^{n}\left(\hat{f}(i, j)-\mu_{0}(i, j)\right)^{2}\right\}
$$

In equation $4, \mu_{0}(i, j)$ represents the pixel value of noise image $\mu_{0}$ at $(i, j)$. The larger the IEF value, the better the denoising algorithm can protect the image edge. PSNR is the ratio between the maximum possible power of the signal and the noise power, and its calculation equation is:

$P S N R=10 \cdot \log _{10}\left(\frac{\left(2^{L}-1\right)^{2}}{\left(\frac{1}{m \cdot n} \sum_{i=1}^{m} \sum_{j=1}^{n}(\hat{f}(i, j)-f(i, j))^{2}\right)}\right)$

In equation $5, \mathrm{~L}$ represents the maximum gray level. The larger the PSNR value, the higher the image quality and the less noise it contains, that is, the better the image denoising algorithm is. SSIM mainly extracts the structural information through the human vision system, and then reflects the attributes of the overall image structure. The calculation equation is as follows:

$$
\left\{\begin{array}{l}
\operatorname{SSIM}(I, R)=(l(I, R))^{\alpha}(c(I, R))^{\beta}(s(I, R))^{\gamma} \\
l(D, R)=\frac{2 \mu_{D} \mu_{R}+C_{1}}{\mu_{D}^{2}+\mu_{R}^{2}+C_{1}} \\
c(D, R)=\frac{2 \sigma_{D} \sigma_{R}+C_{2}}{\sigma_{D}^{2}+\sigma_{R}^{2}+C_{2}} \\
s(D, R)=\frac{\sigma_{D R}+C_{3}}{\sigma_{D} \sigma_{R}+C_{3}}
\end{array}\right.
$$

In equation $6, \alpha, \beta, \gamma$ are the weight of $\mathrm{l}(\mathrm{I}, \mathrm{R}), \mathrm{c}(\mathrm{I}, \mathrm{R}), \mathrm{s}(\mathrm{I}, \mathrm{R}) ; \mathrm{C}_{1}, \mathrm{C}_{2}$, and $\mathrm{C}_{3}$ are three constants. The higher the SSIM value, the higher the image quality.

\subsection{Construction of multi-layer convolutional neural network and image denoising model}

Convolutional neural networks (CNN) is a deep neural network composed of weights and biases of learning and is also the first truly successful algorithm that can train multi-layer neural networks. In general, the unit layer in the $\mathrm{CNN}$ structure is mainly convolutional layer and pooling layer, and each convolutional layer can be connected to a pooling layer, in which the convolutional layer mainly carries out feature extraction. The pooling layer is based on the principle of local correlation, which integrates the feature points in the small field and obtains new features. The common structure diagram of convolutional layer and pooling layer is shown in Figure 1, which includes the feature layer of $n$ layer and is composed of the input feature surface and the output feature surface (convolutional layer and pooling layer) respectively, in which the pooling layer is composed of a convolutional layer.

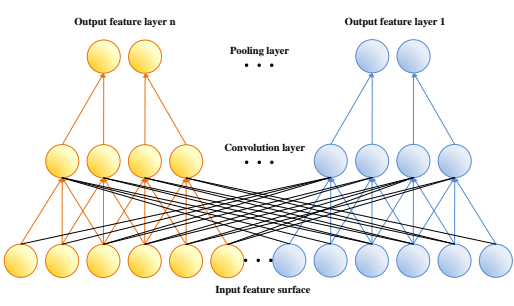

Figure 1: The common structure diagram of the convolutional layer and pooling layer

Compared with the fully connected neural network, CNN has the characteristics of local perception and weight sharing. Moreover, the CNN training process is a supervised learning process, and the flow chart of the training is shown in Figure 2. After CNN starts training, it needs to initialize the network now. Then, through forwarding propagation, the convolution nucleon begins to collect samples. After the samples are output, the loss is calculated, and the backpropagation is carried out to update the weights. If all samples have been trained, the whole training will be completed; If the training is not completed, it should be returned to the total training samples and labels. Untrained samples are extracted and then sampled until all samples are trained. Before the start of the training, a small random number should be selected to ensure that CNN would not reach a saturation state due to too large parameters at the beginning of the training.

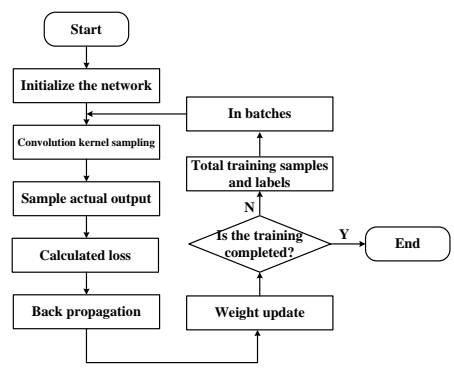

Figure 2: The training process of multi-layer convolutional neural network

In order to make up for the shortcomings of the previous CNN model, the model of MP-CNN is designed to remove the noise in the image. First, the 
complete image needs to be input into $\mathrm{CNN}$ to de-noising, so as to get a clean image. Assuming that the image containing noise is $\mathrm{X}$, then the calculation formula of the extraction layer of multiple features is as follows:

$F=\operatorname{concat}\left[\begin{array}{l}\max \left(0, \sum_{d=1}^{64} \omega_{1 \times 1}^{1} \cdot x+b^{1}\right), \max \left(0, \sum_{d=1}^{64} \omega_{3 \times 3}^{2} \cdot \max \left(0, \sum_{d=1}^{64} \omega_{1 \times 1}^{2,1} \cdot x+b^{2,1}\right)+b^{2}\right), \\ \max \left(0, \sum_{d=1}^{64} \omega_{5 \times 5}^{3} \cdot \max \left(0, \sum_{d=1}^{64} \omega_{1 \times 1}^{3,1} \cdot x+b^{3,1}\right)+b^{3}\right), \max \left(0, \sum_{d=1}^{64} \omega_{7 \times 7}^{4} \cdot x+b^{4}\right)\end{array}\right]$

In equation $7, \omega$ represents the weight, $b$ is on behalf of the bias, subscript of $\omega$ represents the size of the convolution nucleon, index value of $b$ represents the number of layers of the network, $d$ represents the number of channels, $\mathrm{F}$ is the output value of the multi-scale feature extraction layer (that is, the input value of the parallel structure). Then the calculation formula of the output clear image is:

$y=x-\omega_{3 \times 3}^{27} \cdot \max \left(0, \sum_{d=1}^{64} \omega_{3 \times 3}^{26} \cdot \operatorname{concat}\left(R_{250}, \mathrm{R}_{10}\right)+b^{26}\right)+b^{27}$

In equation $8, R_{250}$ is the output value of the deep channel, and $R_{10}$ is the output value of the shallow channel.

\subsection{Verification of functional action image denoising model}

In order to verify the denoising effect of a multi-scale parallel convolutional neural network model on functional action images, 100 grayscale images are selected to be constructed as the training data set. In addition, these images are randomly flipped and rotated, and the moving step size is set to 10 . The test set uses 5 images to test the model, and the training set doesn't include the images in the test set, so as to better reflect the generalization ability of the image denoising model. Some of the parameters in the build model need to be set before formal validation can begin. The deep learning framework used in the study is Keras, and the validation tests are all conducted on PC. Xavier is used to initialize the weighted parameters of the convolution nucleon with uniform distribution, and the bias value is set to 0 . In order to ensure the optimization of the model, Adam optimizer is selected to adjust the learning rate, and the initial learning rate is 0.001 , which would decrease with the increase of training times of the model.

\section{RESULTS}

\subsection{The feasibility results of the model MP-CNN}

In order to verify the feasibility of the MP-CNN image denoising Model of the shallow channel and deep channel convolutional layer proposed in this study, 100,000 40*40 images are used to train Model-1 (containing only 7 convolutional layers and activation layers), Model-2 (containing only 25 convolutional layers and activation layers) and MP-CNN. Moreover, the noise level of the picture is set as 15,25 and 50 to obtain the noise removal model of the picture at different noise levels. Then the test set is used to compare the PSNR and SSIM of each model, and the results are shown in Figure 3. The PSNR and SSIM values of MP-CNN at different noise levels are greater than those of Model-1 and Model-2.

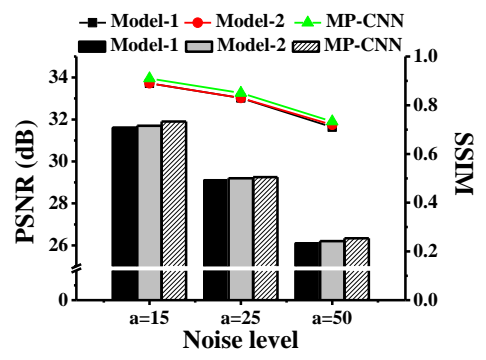

Figure 3: PSNR and SSIM results of noise model with different noise levels

During the test, the difference between the mean MSE values of the three models at different noise levels is calculated, and the results are shown in
Figure 4. It can be concluded that the MSE values of the MP-CNN model are smaller at different noise levels.

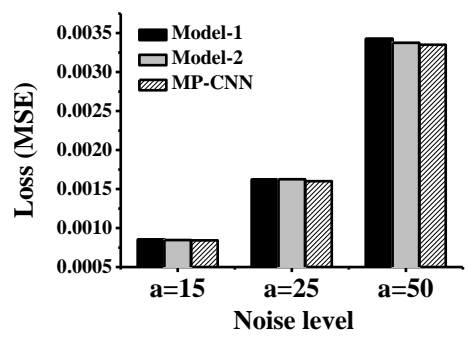

Figure 4: MSE results of noise model at different noise levels

\subsection{Objective evaluation of index results of model MP-CNN}

To verify the denoising feasibility of MP-CNN model designed in this study, WNNM, EPLL, TNRD, DnCNN-S and SCNN models are selected for comparison. Finally, PSRN and SSIM values are used to compare the effect of image denoising. The larger the PSNR value, the lower the distortion rate after image denoising; the larger the SSIM value, the higher the similarity between image and original image after image denoising. Firstly, the differences between PSRN values of this model at noise levels of 15, 25 and 50 are compared. The results are shown in Figure 5. It can be concluded from Figure $5 \mathrm{~A}$ that the PSNR value of MP-CNN model is the highest at different noise levels. According to Figure $5 \mathrm{~B}$, it can be concluded that the test time of DnCNN-S, SCNN, and MP-CNN models is short, and there is little difference between groups.
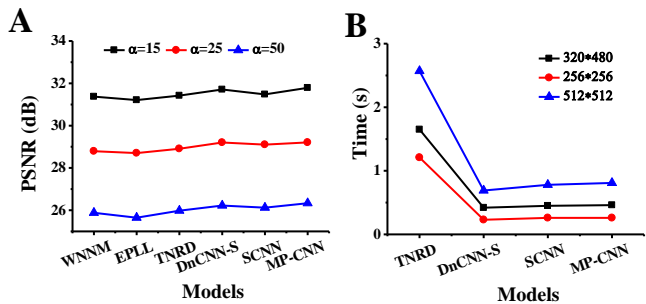

Figure 5: Comparison of PSNR value and test time of noise model at different noise levels

\subsection{Subjective evaluation index results of model MP-CNN}

A picture in the data set is taken as an example. After processing it with 25 noise, the denoising effects of WNNM, TNRD, DnCNN-S and MP-CNN models are compared respectively. As shown in Figure 6, Figure 6A is the original grayscale image; Figure 6B is the image after noise processing, which shows that the image is blurred and unreal, and the man's facial features in the image can no longer be seen clearly. Figures 6C and 6D show the denoising effect of the WNNM and TNRD models, respectively. It shows that these two models can remove most of the noise in the image well, but the processing effect of the facial feature noise in the relatively fine men in the image is poor. Figure 6E shows the noise removal effect of the DnCNN-S model. It shows that the man's facial features have a general outline and are relatively clear, but the muscle lines of the man's arm and the details of the hand image are not as good as those of the MP-CNN model in Figure 6F.

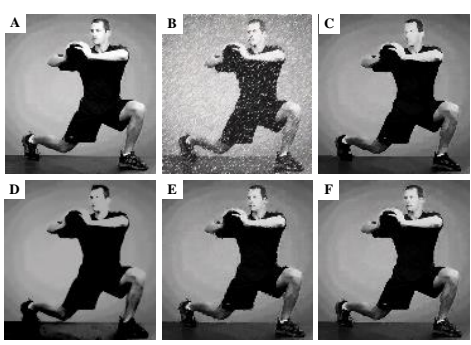

Figure 6: Subjective evaluation results of denoising effects of different models 


\section{DISCUSSION}

Noise reduction processing is a basic operation to enhance image quality, and studies have shown that PSNR, MSE, and SSIM can be used to evaluate the performance of image noise reduction processing [7]. Under different noise levels, the PSNR and SSIM values of the MP-CNN model with two channels are higher than those of the other models, indicating that the MPCNN model has a better effect on noise removal in images and better feasibility. The PSRN values of MP-CNN model are all larger than WNNM, EPLL, TNRD, DnCNN-S and SCNN models, indicating that MP-CNN had the best effect on noise removal in images. In a study showed that SIMM value was used to improve the filter and found that noise in the image could be removed better [8]. Some researchers used PSNR, MSE and SSIM to carry out a qualitative and quantitative comparison of image indicators [9]. The convolutional neural network is one of the representative algorithms of deep learning, and it has the ability to represent learning. The MSE value of the MP-CNN model is small, indicating that the MP-CNN model has a high similarity with the original image after noise removal. The subjective results show that the MP-CNN model can make full use of the features of the image, so as to retain the details and edge information of the image. Compared with other image denoising models, the MP-CNN model can significantly improve the similarity of structures in the image and the effect of noise removal. A group researcher proposed a new three-channel image feature extraction method based on the convolutional neural network and found that the accuracy and efficiency of the calculation were effectively improved [10]. Some of researcehrs found that the multi-layer perceptron and convolutional neural network were jointly constructed for image classification, and the results showed that the optimal image classification could be achieved [11]. The selection of image fusion requires the selection of appropriate evaluation indexes, which are mainly divided into the subjective evaluation and objective evaluation, and the correlation between the comprehensive index and subjective evaluation indexes is higher than that between individual evaluation indexes and evaluation results [12]. However, it is found that the subjective evaluation results of MP-CNN are all better than other models, indicating that this model has a better effect on noise removal in images.

\section{CONCLUSION}

To ensure the image protection during the acquisition and transmission of functional action images, an image denoising model based on a multi-scale parallel convolutional neural network is proposed. The results show that the model can improve the effect of image denoising and speed up the convergence. However, due to the limited number of images included in the training and test in this study, it is necessary to supplement the sample size to conduct verification tests again and study its application in video image segmentation. In a word, the results of this study can lay a foundation for the application of the convolutional neural network in image denoising.

\section{REFERENCES}

[1] Zeng, X., Bian, W., Liu, W. 2015. Dictionary Pair Learning on Grassmann Manifolds for Image Denoising. IEEE Transactions on Image Processing, 24 (11), pp. 4556-4569.

[2] Klosowski, J., Frahm, J. 2017. Image denoising for real-time MRI. Magnetic Resonance in Medicine, 77 (3), pp. 1340-1352.

[3] Lan, R., Zou, H., Pang, C. 2019. Image denoising via deep residual convolutional neural networks. Signal Image and Video Processing, (9), pp. $1-8$.

[4] Syed, U.A., Kunwar, F., Iqbal, M. 2014. Guided Autowave Pulse Coupled Neural Network (GAPCNN) based real time path planning and an obstacle avoidance scheme for mobile robots. Robotics and Autonomous Systems, 62 (4), pp. 474-486.

[5] Zhong, Y., Liu, L., Zhao, D. 2019. A generative adversarial network for image denoising. Multimedia Tools and Applications, (2), pp. 1-13.

[6] Shan, H., Zhang, Y., Yang, Q. 2018. 3D Convolutional Encoder-Decoder Network for Low-Dose CT via Transfer Learning from a 2D Trained Network. IEEE Transactions on Medical Imaging, 37(6), pp. 1522.

[7] Mathew, J., Zollanvari, A., James, A.P. 2017. Edge-Aware Spatial Denoising Filtering Based on a Psychological Model of Stimulus Similarity. IEEE Access, 6, pp. 3433-3447.

[8] Hasan, M., El-Sakka, M.R. 2018. Improved BM3D image denoising using SSIM-optimized Wiener filter. EURASIP Journal on Image and Video Processing, (1), pp. 25.

[9] Saadia, A., Rashdi, A. 2018. Incorporating fractional calculus in echocardiographic image denoising. Computers \& Electrical Engineering, 67, pp. 134-144.

[10] Liu, S., Liu, Z. 2017. Multi-Channel CNN-based Object Detection for Enhanced Situation Awareness. 30, pp. 1-9.

[11] Zhang, C., Pan, X., Zhang, S. 2017. A rough set decision tree based MLP-CNN for very high resolution remotely sensed image classification. ISPRS-International Archives of the Photogrammetry, Remote Sensing and Spatial Information Sciences, pp. 1451-1454.

Han, Z., Lin, S. 2017. Multiband fusion image evaluation method based on correlation between subject and object evaluation. Opto-Electronic Engineering, 44 (9), pp. 895-902. 